\title{
Yahoo!Search and Web API Utilized Mashup based e-Leaning Content Search Engine for Mobile Learning
}

\author{
Kohei Arai 1 \\ Graduate School of Science and Engineering \\ Saga University \\ Saga City, Japan
}

\begin{abstract}
Mashup based content search engine for mobile devices is proposed. Mashup technology is defined as search engine with plural different APIs. Mash-up has not only the plural APIs, but also the following specific features, (1) it enables classifications of the contents in concern by using web 2.0 , (2) it may use API from the different sites, (3) it allows information retrievals from both sides of client and server, (4) it may search contents as an arbitrary structured hybrid content which is mixed content formed with the individual content from the different sites, (5) it enabling to utilize REST, RSS, Atom, etc. which are formed from XML conversions. The mash-up should be a flexible search engine for any purposes of content retrievals. The proposed search system allows 3D space display of search menus with these peculiarities on Android devices. The proposed search system featuring Yahoo!search BOSS and Web API is also applied for e-learning content retrievals. It is confirmed that the system can be used for search a variety of elearning content in concern efficiently.
\end{abstract}

Keywords-Mashup; API; web 2.0; mobile devices; e-learning content; content retrieval; Yahoo!saerch BOSS; Web API

\section{INTRODUCTION}

E-learning content retrievals with mobile terminals have not been done easily due to the fact that display size is not good enough for search as well as there are a variety of contents types, video, document, etc. On the other hand, mashup technology [1], [2] supported web 2.0 [3] allows gathering information of e-learning contents efficiently and effectively. Meanwhile, visualization of web contents has been well developed. Therefore, e-learning content search engine can be improved in terms of efficiently and effectively based on the aforementioned mashup technology and visualization tools 1 .

Web API [4] based e-learning content search engine is developed with Yahoo!search BOSS (Build your Own Search Service) [5] which is called ELDOXEA: E-Learning Content Search Engine [6]-[10]. Not only major keyword, but also minor keywords derived from the descriptions and keywords in the header information of URL which is hit by the first major keyword are used for search. The search engine proposed here is ELDOXEA for mobile devices. One of the disadvantages of mobile devices is relatively small size of display. Therefore, in particular, representation method for search results has to be considered for fitting the search result contents efficiently and effectively.

There are many web visualization tools. In particular, UNIX software of Natto view [11] and mashup based search engine with www visualization of Flowser ${ }^{2}$ are sophisticated tools. With the reference to these software tools, the proposed e-learning content search engine is designed.

E-learning content search engine with web API based approach is proposed in the following section. Bing and Google API are additionally featured. Furthermore, thesaurus based search results prioritized method is featured. Application software tool for the search engine is described followed by implementation and some experimental results. Then concluding remarks are described with some discussions.

\section{PROPOSED METHOD AND SYSTEM}

\section{A. Mashup Technology}

Mashup is defined as a method for providing web services and applications based on combination of more than two web APIs. It allows improve usability by combining search results which are obtained from the different web APIs separately. Search results from web APIs, in general, in the form of XML or JSON [12]. Therefore, it is easy to combine the search results into one. The following four web APIs are used for the proposed search engine,

1) Yahoo!search: web URL search API http://search.yahooapis.jp/PremiumWebSearchService/V1/we bSearch

2) Yahoo!search: Image retrieval API http://search.yahooapis.jp/PremiumImageSearchService/V1/i mageSearch

3) Youtube Data API developed by Google Inc. http://gdata.youtube.com/feeds/api/videos

4) Product Advertising API developed by Amazon.com, Inc.

http://ecs.amazonaws.jp/onca/xml

\footnotetext{
${ }^{2}$ http://www.flowser.com/
}

https://supportcenter.checkpoint.com/supportcenter/portal?eventSubmit_doGo viewsolutiondetails $=\&$ solutionid $=$ sk 30765 


\section{B. Search Result Display}

In order to display search results in a comprehensive manner, the following four candidate models, helix, star, star helix and star slide are trade-off.

One of important roles of the displaying search results is to show the priority of the results. From the top to bottom priority, "helix model" of displaying method allows priority representation from the top to the bottom of the display positions in a spiral order. It, however, is not appropriate displaying method because there are many types of the search results, document, URL, image, etc. Therefore, it is complicated and overlapped so much among the search result types.

Another model of the search result representation is "star model". This model is adopted by Flowser. The search results are classified by content types of nodes. From the top node to the node in concern, the length of rink between both shows priority. It, however, is not so easy to see because the nodes are located in the 3D domain of the display positions.

Star-Helix model combines the aforementioned two models, star and helix models. It, however, is still difficult to see the priority. Star-Slide model of search result display method is the most appropriate model as shown in Fig.1.

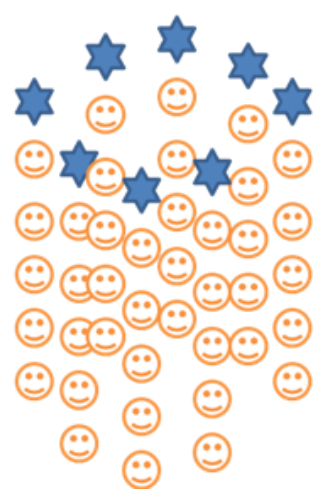

Fig. 1. Star-Slide Model

In the Fig.1, star shows search result types while smile marks shows contents (URLs). From the top to bottom priority, "star-slide model" of displaying method allows priority representation from the top to the bottom of the display positions. It has to be displayed onto mobile device displays in a 3D CG representation. Away3D [9] is used for this purpose. There are a plenty of 3D CG representation software tools, OpenGL, ADOBE AIR, etc. under the programming language of Java, Objective-C, etc. In particular, Away3D is used on the ADOBE AIR (Stage3D) of Action Script as a library. The geometric relation between camera and object is shown in Figure 2 as well as rendering of object together with staging device.

\section{LEDOXEA}

LEDOXEA is mobile devices version of ELDOXEA: ELearning Content Search Engine. After the activation of LEDOXEA, initial image of start-up is displayed onto mobile device display as shown in Fig.3 (a). Immediately after this, key-in is available with screen keyboard as shown in Fig.3 (b).

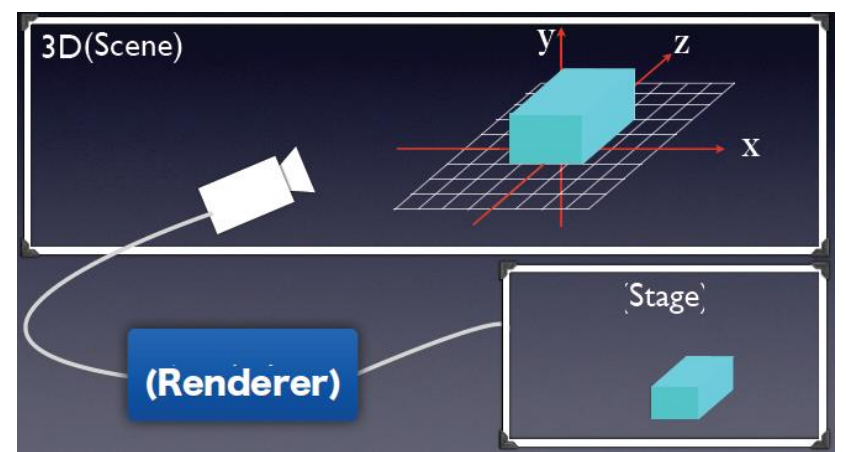

Fig. 2. Geometric relation among 3D view port, camera, object and 3D area definition for rendering

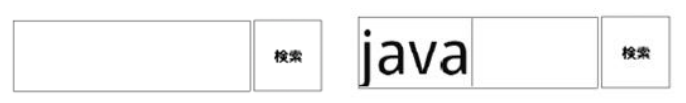

Title

Discription

(a) Initial image

Fig. 3. Display image

When users key-in their keyword in the dialog box as shown in Fig.4 (a), then users press the search button for retrievals. After that, users get their search results as shown in Fig.4 (b).

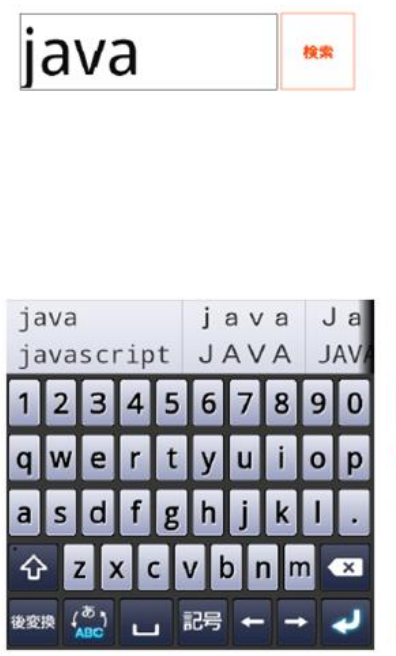

(a) Search initiate image
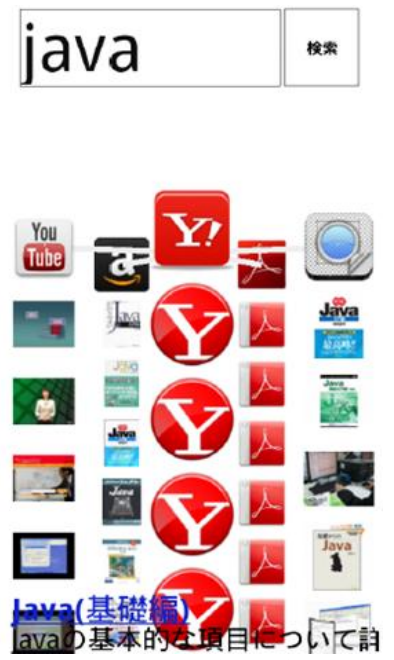

(b) Search results
Fig. 4. Display image

In this case, five content types and icons of search results of URLs appear in order of their priorities. Five content types 
are (1) search engines such as Google, Yahoo search, (2) moving picture contents such as YouTube, (3) document contents, (4) image contents and (5) general purpose content search such as Amazon.com which are shown in Fig.5.

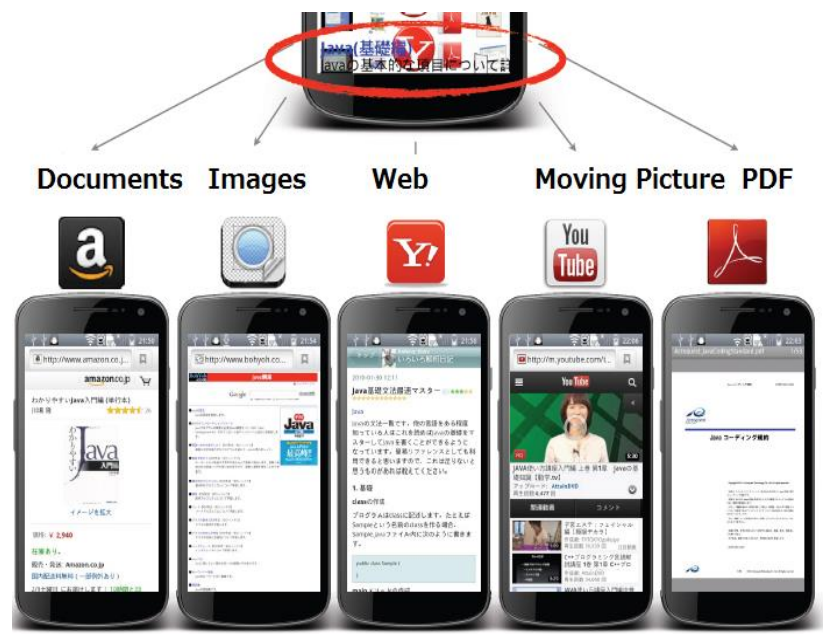

Fig. 5. Content types for search

Search results can be moved up and down when users swipe in the vertical direction as shown in Fig.6 (a). Therefore, search result content can be seen one by one. When users would like to see other types of search results of contents, they have to swipe the display in the horizontal direction as shown in Fig.6 (b).

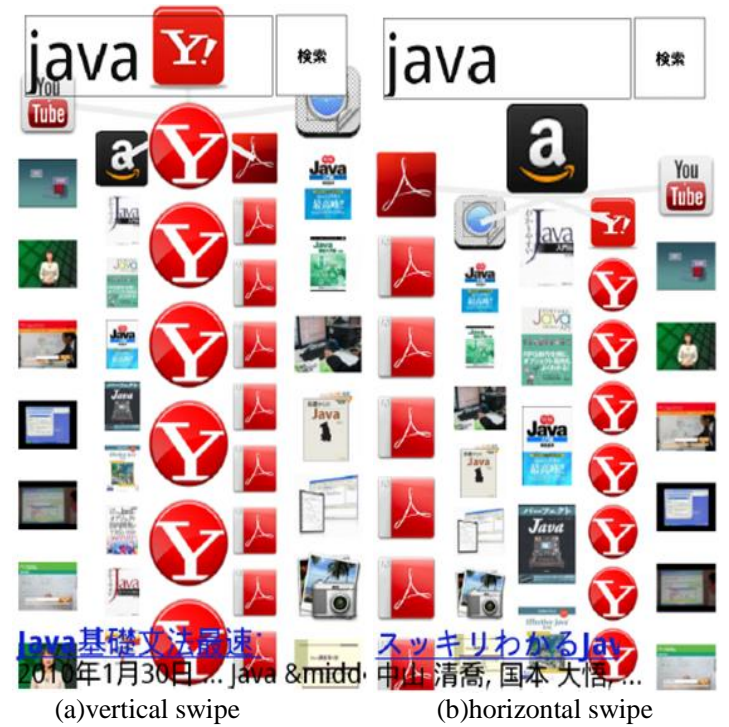

Fig. 6. Move up and down as well as ration with vertical swipe and horizontal swipe

The icon sizes are different by content type for representation of the search results in 3D space on the display as shown in Fig.7. When users tap the title summary which is located the bottom of the display as shown in Fig.8, then users may take a look at the search result of contents through browser or external applications. There is "back button" for getting back to the previous display image after the referencing the search result contents.

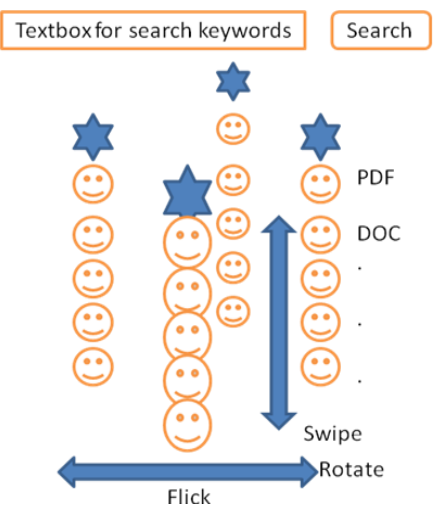

Fig. 7. 3D display of the icon during flick, swipe, and rotational operations

Search content types are rotated when users swipe the content type icons. These rotation angles can be calculated as shown in Fig.8.

Icon operations with swipe actions are illustrated in Fig.9. Swipe action in the vertical direction allows search results candidate selections. Meanwhile, swipe action in the horizontal direction allows content type selections. These can be done through camera position changes in $3 \mathrm{D}$ space in the CG space of Away 3D.

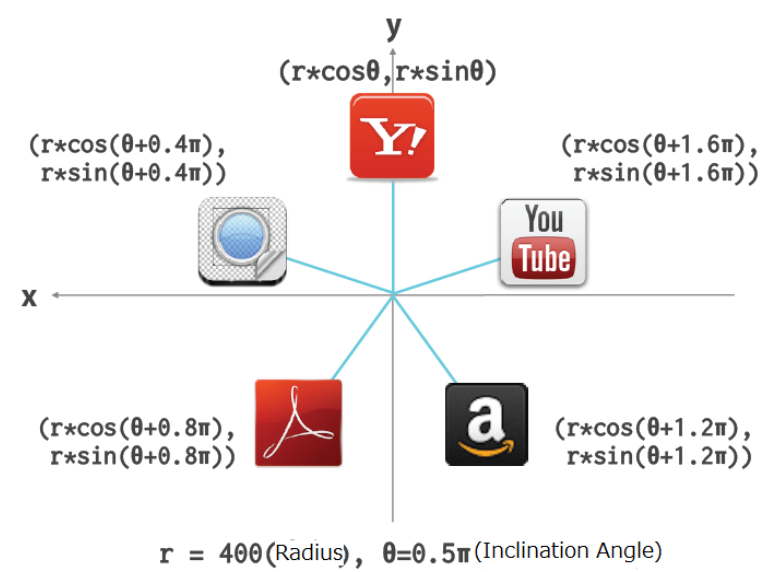

Fig. 8. Rotation angle of the five icons

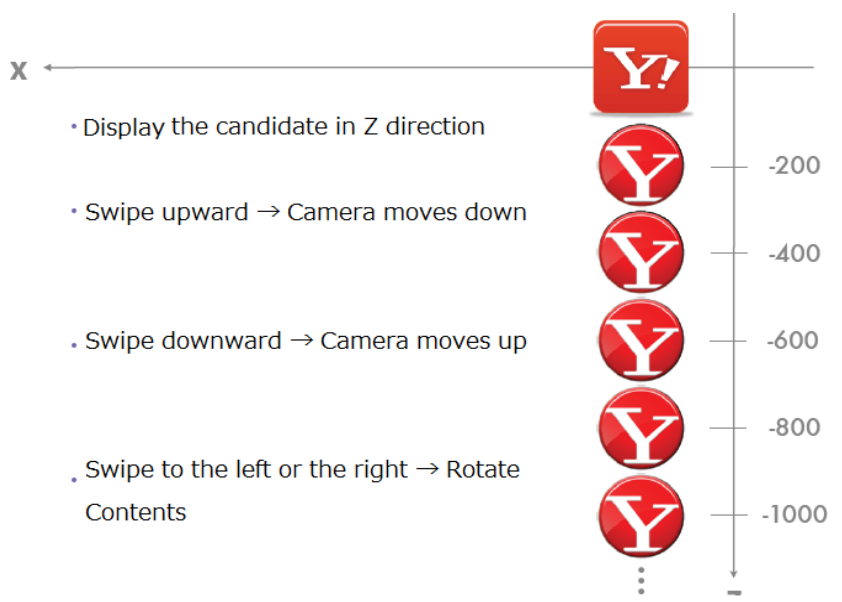

Fig. 9. icon manipulations utilizing swipe operations 


\section{A. LEDOXEA version 2}

From the web site stability or availability points of view, the proposed search engine employed Bing API instead of Yahoo!Search. Display image layout is shown in Fig. 10.

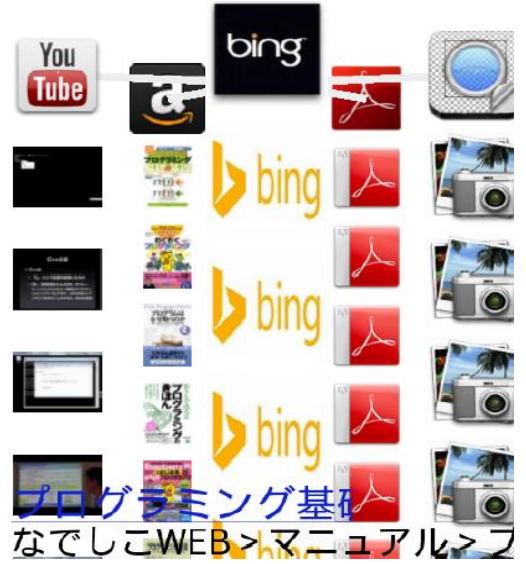

Fig. 10. Example of search result display with Bing API based LEDOXEA

YouTube (moving pictures), Amazon site, ADOBE content (documents), and Image contents are available for search. These are common to the previous LEDOXEA featuring Yahoo!Search. Search results are aligned in the order of their priority. When users choose one of the icon of search result, then the hyperlink of the search result appears on the bottom of the screen. Also, users get the contents when they click the hyperlinked search result. This is referred to the version 1 of LEDOXEA hereafter.

\section{B. Thesaurus Approach}

In order to improve effectiveness of search engine, not only major keyword but also minor keywords can be used for e-learning content search. In addition to the dialog box, thesaurus search is available for the LEDOXEA version 2. Example of the keyword input image display is shown in Fig.11 (a). There is the dialog box for thesaurus search which is situated just under the dialog box for the major keyword. Example of the image of thesaurus search results is shown in Fig.11 (b).
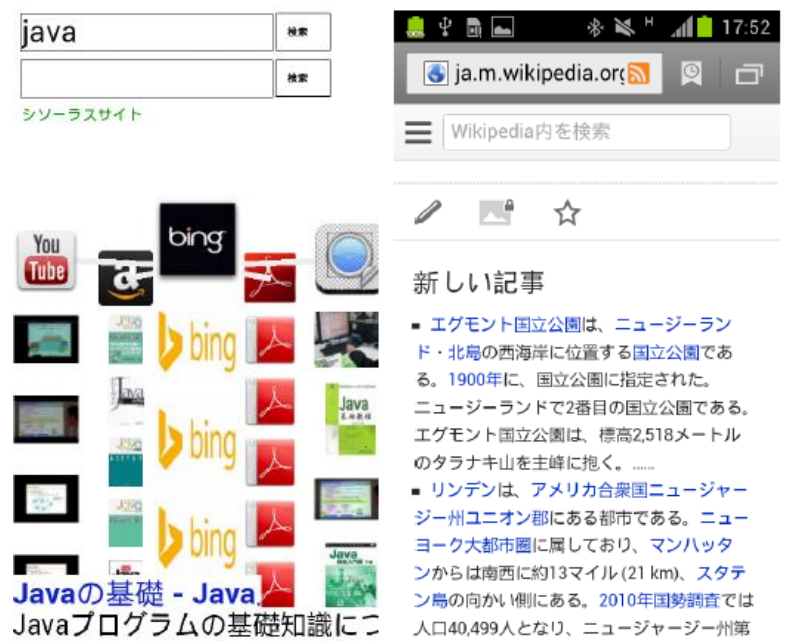

(a)Thesaurus search engine

(b)Example of thesaurus search result
In this example, "Wikipedia" of thesaurus search engine is selected. Appropriate minor keywords are available as the thesaurus search results. Therefore, much appropriate elearning content search can be done with major and minor keywords.

Fig.12 (a) shows the other thesaurus search result by keyword types abstract, history, word, footnote, reference, related site, other link are available for thesaurus search. Also, several languages are available for search by clicking the bottom button. Fig.12 (b) shows an example of e-learning content search with the major keyword of "Java". When users key-in the minor keyword of “文法” (grammar in English) in the dialog box of thesaurus search engine, then they can get the much appropriate search results rather than LEDOXEA version 1 (without thesaurus engine).

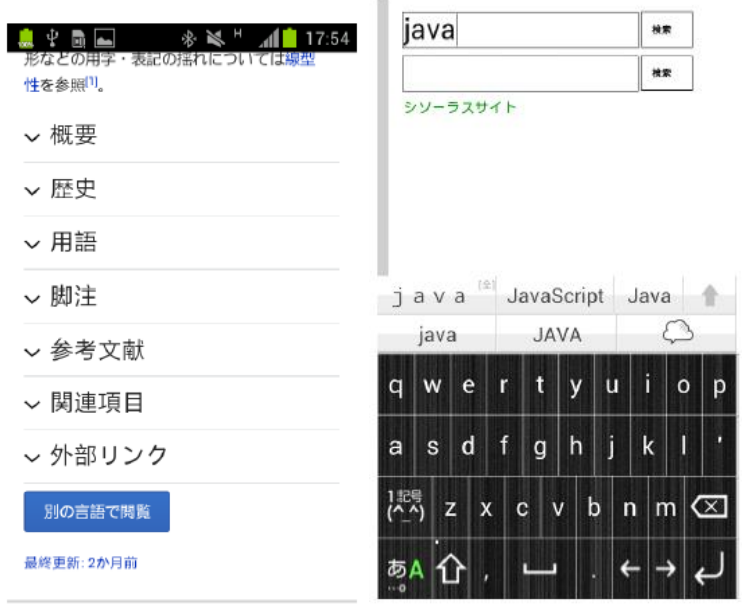

(a)Thesaurus search keyword (b)Example for "Java"

Fig. 12. Search keyword types selection for thesaurus search and example of e-learning content search with the major keyword of "Java"

Fig.13 (a) shows an example of search image when users key-in the major keyword "Java" and the minor keyword "grammar" for the thesaurus search engine while Fig.13 (b) shows an example of search image when users key-in the major keyword "Java" and the minor keyword "question and answer" for the thesaurus search engine.

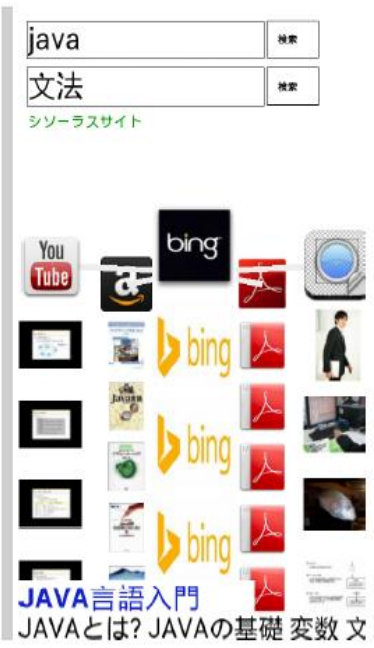

(a) minor keyword "grammar"

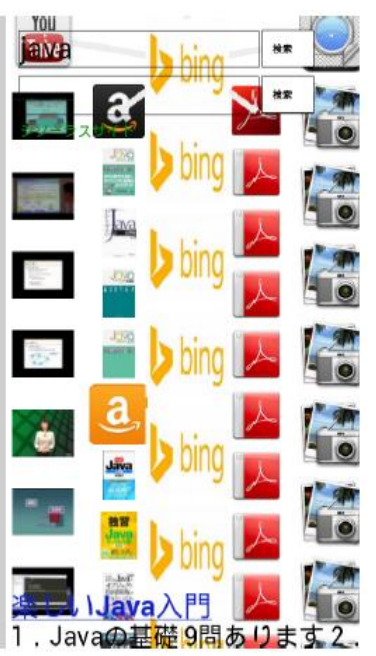

(b) minor keyword "question and answer" 


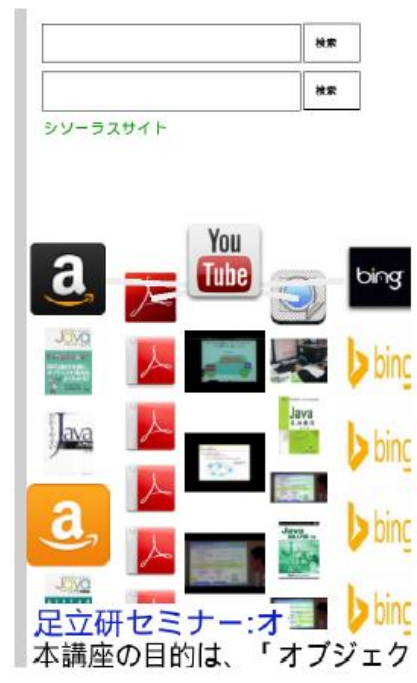

(c)minor keyword "lecture"

Fig. 13. Examples of e-learning content search results with major keyword of "Java" and minor keywords, "grammar" and "Question and answer" as well as "Lecture" for thesaurus search results

On the other hand, Fig.13 (c) shows an example of search image when users key-in the major keyword "Java" and the minor keyword "question and answer" for the thesaurus search engine.

Thus users can take a look at the search results from the top to the bottom priority of the search results by content type by type. Also, the highest priority of search result appears at the bottom of the image with hyperlink. In this example, the book on Java language specifically programming grammar is greatly featured is shown as search result. Users may input minor keywords on their own. Users also may use thesaurus search engine. It is totally up to users.

\section{EXPERIMENTS}

\section{A. Implementation}

Implementation of the proposed software is done with FlashProfessionalCS6 on Android 4.0.3. In order to publicize of the implemented source code based on Action Script, the following library, framework, image files are executed,

1) Away3D 4.1.0 Alpha (away3d-core-fp11 $4 \quad 1 \quad 0$
Alpha.swc)[8]
2) Bulk Loader(bulk loader.swc)[13]
3) As3 Crypto (as3crypto.swc)[14
4) norm-top.png (128x128px of png file)
5) norm.png (128x128px of png file)
6) img-top.png (128x128px of png file)
7) img.png (128x128px of png file)
8) doc-top.png (128x128px of png file)
9) doc.png (128x128px of png file)
10) mv-top.png (128x128px of png file)
11)ama-top.png (128x128px of png file)
12)ama.png (128x128px of png file)
13)defImgXML.xml (XML file which are acquired with Y!
Image Retrieval API)

These source code package are provided by the URL of Github

https://github.com/legnoh/ledoxea

\author{
A.1 Main.as(ActionScript3.0) \\ package \{ \\ import flash.display.BitmapData; \\ import flash.display.Sprite; \\ import flash.display.SimpleButton; \\ import flash.events.*; \\ import flash.filesystem.StorageVolumeInfo; \\ import flash.geom.Matrix; \\ import flash.geom.Vector3D; \\ import flash.text.*; \\ import flash.utils.ByteArray; \\ import flash.utils.escapeMultiByte; \\ import flash.net.URLLoader; \\ import flash.net.URLRequest; \\ import flash.ui.Keyboard; \\ import flash.desktop.NativeApplication; \\ import away3d.containers. *; \\ import away3d.entities.*; \\ import away3d.materials.TextureMaterial; \\ import away3d.primitives.*;
}

On the other hand, XML file is created which is defined Android application software. Meanwhile, used APIs are Yahoo!JAPAN Web Retrieval API,Yahoo!JAPAN Image Retrieval API,YouTube Data API, and Amazon Product Advertising API.

\section{B. Experiments}

Fig.14 shows an example of display image of smartphone of which the proposed search engine is implemented (github.com/legnoh/ledoxea).

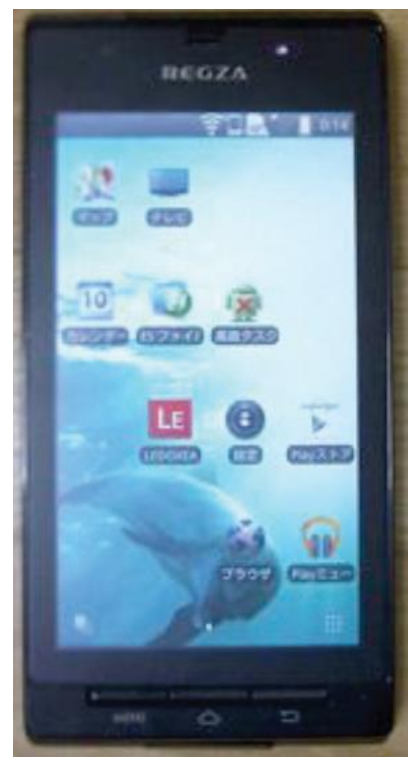

Fig. 14. Example of display image of smartphone of which the proposed search engine is implemented (github.com/legnoh/ledoxea)

"LE" of icon denotes LEDOXEA. 


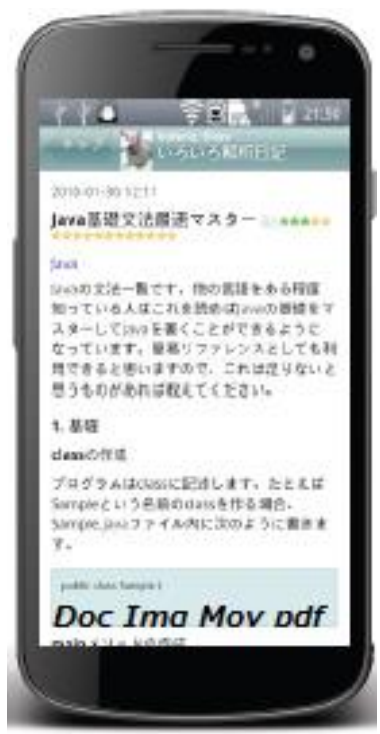

Fig. 15. Example of linked retrieved results (Merged contents of Yahoo search results with the other retrieved contents)

Twenty of university students (information science department) participate the experiments. They use the LEDOXEA and then they evaluate the LEDOXEA. Their comments and suggestions are as follows,

1) It is comfortable to use the LEDOXEA because operability using swipe operations, smooth rendering, usability of Andoroid tablet terminals, WWW visualizations are excellent,

2) Search results can be seen simultaneously,

3) PDF data and YouTube of search results can be accessible from the LEDOXEA directly,

4) Appropriate e-learning contents can be retrieved efficiently with a single keyword.

5) Prioritizing the search results is excellent because the most appropriate e-learning content can be found easily,

6) Five e-learning contents can be seen simultaneously with a single keyword,

7) Still pictures as well as moving pictures are displayed their thumbnail, it is comprehensive.

\section{CONCLUSION}

Mashup based content search engine with mobile terminals is proposed. Mashup technology is defined as search engine with plural different APIs. Mash-up has not only the plural APIs, but also the following specific features. it enables classifications of the contents in concern by using web 2.0, it may use API from the different sites, it allows information retrievals from both sides of client and server, it may search contents as an arbitrary structured hybrid contents which is mixed contents formed with the individual contents from the different sites, it enabling to utilize REST, RSS, Atom, etc. which are formed from XML conversions.

Although mashup allows content search which is same as portal, mashup has the aforementioned different features from portal. Therefore, mash-up is possible to create more flexible search engine for any purposes of content retrievals. The search system which is proposed here is that make it possible to control the graph in the 3D space display with these peculiarity on Android devices. The proposed search system is applied for e-learning content retrievals. It is confirmed that the system enables to search a variety of content in concern efficiently.

It is found that users can take a look at the search results from the top to the bottom priority of the search results by content type by type. Also, the highest priority of search result appears at the bottom of the image with hyperlink. In this example, the book on Java language specifically programming grammar is greatly featured is shown as search result. Users may input minor keywords on their own. Users also may use thesaurus search engine. It is totally up to users.

\section{ACKNOWLEDGMENT}

The author would like to thank Mr. Ryoma Kai and Mr. Yohei Nishiguchi for their effort to the implementation and the experiment.

\section{REFERENCES}

[1] Ahmet Soylu, Felix Mödritscher, Fridolin Wild, Patrick De Causmaecker, Piet Desmet. 2012 . "Mashups by Orchestration and Widget-based Personal Environments: Key Challenges, Solution Strategies, and an Application." Program: Electronic Library and Information Systems 46 (4): 383-428.

[2] Endres-Niggemeyer, Brigitte ed. 2013. Semantic Mashups. Intelligent Reuse of Web Resources. Springer. ISBN 978-3-642-36402-0

[3] O'Reilly, T., 2005. What is Web 2.0. Design Patterns and Business Models for the Next Generation of Software, p. 30

[4] Benslimane, D.; Dustdar, S.; Sheth, A. (2008). "Services Mashups: The New Generation of Web Applications". IEEE Internet Computing 10 (5): 13-15. doi:10.1109/MIC.2008.110

[5] Tuesday, August 17th, 2010 (2010-08-17). "As Bing Takes Over Yahoo Search, SearchMonkey Dies, BOSS Is No Longer Free, But Site Explorer Still Works". TechCrunch. Retrieved 2012-09-18.

[6] Kohei Arai, Mash-up based content search engine for mobile devices, International Journal of Advanced Research in Artificial Intelligence, 2, 5, 39-43, 2013.

[7] Kohei Arai and Tolle Herman, Module based content adaptation of composite e-learning content for delivering to mobile learners, International Journal of Computer Theory and Engineering, 3, 3, 382387, 2011.

[8] Kohei Arai, Herman Tolle, Efficiency improvements of e-learning document search engine for mobile browser, International Journal of Research and Reviews on Computer Science, 2, 6, 1287-1291, 2011.

[9] K.Arai, T.Herman, Efficiency improvement of e-learning document search engine for mobile browser, International Journal of Research and review on Computer Science, 2, 6, 1287-1291, 2012.

[10] K.Arai, T.Herman, Video searching optimization with supplemental semantic keyword for e-learning video searching, International Journal of Research and Review on Computer Science, 3, 3, 1640-1644, 2012.

[11] H. Shiozawa, K. Okada, Y. Matsushita: 3D Interactive Visualization for Inter-Cell Dependencies of Spreadsheets, Proc. IEEE InfoVis '99, pp. 79-82, Oct. 1999.

[12] Douglas Crockford (July 2006). "IANA Considerations". The application/json Media Type for JavaScript Object Notation (JSON). IETF. sec. 6. RFC 4627. https://tools.ietf.org/html/rfc4627\#section-6. Retrieved October 21, 2009.

\section{AUTHORS PROFILE}

Kohei Aarai He received BS, MS and PhD degrees in 1972, 1974 and 1982, respectively. He was with The Institute for Industrial Science and Technology of the University of Tokyo from April 1974 to December 1978 and also was with National Space Development Agency of Japan from 
January, 1979 to March, 1990. During from 1985 to 1987, he was with Canada Centre for Remote Sensing as a Post Doctoral Fellow of National Science and Engineering Research Council of Canada. He moved to Saga University as a Professor in Department of Information Science on April 1990. He was a councilor for the Aeronautics and Space related to the Technology Committee of the Ministry of Science and Technology during from 1998 to
2000. He was a councilor of Saga University for 2002 and 2003. He also was an executive councilor for the Remote Sensing Society of Japan for 2003 to 2005. He is an Adjunct Professor of University of Arizona, USA since 1998. $\mathrm{He}$ also is Vice Chairman of the Commission-A of ICSU/COSPAR since 2008. He wrote 33 books and published 500 journal papers. 